\title{
Adapting the Bass Guitar for One-Handed Playing
}

\author{
Harrison, Jacob \\ Centre for Digital Music \\ Queen Mary University of London \\ London \\ UK \\ j.harrison@qmul .ac.uk
}

\author{
McPherson, Andrew P. \\ Centre for Digital Music \\ Queen Mary University of London \\ London \\ UK \\ a.mcpherson@qmul.ac.uk
}

June 30, 2017

\begin{abstract}
This paper presents a prototype system for adapting the bass guitar for one-handed musicians. We discuss existing solutions to accessible musical instruments, followed by the results of an online survey of bass guitarists, which informed the design of a prototype bass guitar adaptation. The adaptation comprises a foot-operated MIDI controller with a solenoid-actuated fretting mechanism, providing access to six frets across two strings of the bass. A study involving six bassists rehearsing and writing a bass guitar accompaniment with the adapted bass highlighted unexpected facets of bass guitar playing, and provided insights into the design of future accessible string instruments.
\end{abstract}

\section{Keywords}

Instruments, Interfaces, Performance, Social aspects

\section{Introduction}

It is a fact often taken for granted that nearly all musical instruments are designed to be played with both hands at all times. Even for instruments on which it is possible to play one-handed, such as keyboards and some wind instruments, the written repertoire generally requires two hands. For many people, including those with upper limb disabilities, this requirement is prohibitive to involvement in musical performance.

Music therapy practices have demonstrated the health, social and psychological benefits of music making (Nayak et al., 2000; Särkämö et al., 2008; Hunt et al., 2004), and accessible instruments provide a means for those with disabilities to enjoy these benefits. However, many approaches to accessible musical instruments focus on ease of use and a low barrier to expressive music generation (see the $\operatorname{Skoog}^{1}$ and the SoundBeam (Swingler, 1998)). These represent a class of accessible instruments which are designed purely as therapeutic devices, as opposed to fully-realised instruments which allow the user to excel musically.

This paper presents a system for playing bass guitar without the use of one hand and arm. We developed a prototype actuated fretting mechanism for bass guitar with a foot-controlled MIDI interface, to allow for one-handed playing. We then evaluated the system through a performance study, gathering video data and subjective responses, in order to assess the viability of such a system for one-handed bass playing. This uncovered more general insights into the role of the plucking and fretting hands in string instrument performances, and could inform the design of future accessible string instruments.

We present a review of existing approaches to accessible musical instruments, in Section 2. This is followed by a discussion of an online survey of bass guitarists, which we used to inform the design of instrument adaptation (Section 3). The design and implementation of the prototype system is discussed in Section 4, with an evaluation of its performance including subjective responses. Finally in Section 5 we present and discuss the results of a user study, including emergent adaptations to playing technique, and implications for future adapted string instrument designs.

${ }^{1}$ http://skoogmusic.com/ 


\section{Background}

\subsection{Accessible Instruments and Interfaces}

Here, we make the distinction between two categories of instruments designed for people with disabilities: 'therapeutic devices' and 'performance-focused instruments'. The former category refers to instruments which offer a low barrier to expressive music making, allowing non-musicians or those with neuro-disabilities to express themselves without the need for large amounts of practice. These instruments work particularly well in group music making workshop contexts, or as part of music therapy sessions. The latter category refers to instruments which may require a large amount of practice in order to master, but which allow the user a similar level of expression and virtuosity as a traditional instrument would to an able-bodied performer. This category tends to encapsulate bespoke or DIY projects, as the individuals' physical abilities can vary greatly, making for very specific requirements on the interface and mapping strategies. Many projects in this vein involve a participatory design process, in which the end-user is deemed the 'expert' in their own needs and requirements (Lin et al., 2014).

Many prominent examples of accessible instruments (generally in the 'therapeutic devices' category) are given by Larsen et al. (2016). They provide an overview of the use cases of accessible Digital Music Instruments (DMIs), as well as a discussion of two common causes of disability in adults (Cerebral Palsy and stroke). Larsen discusses the emerging practice of Neurologic Music Therapy (NMT) from the point of view of brain plasticity: the process by which the brain can reorganise itself through repeated cognitive processes, such as playing music. This process is the basis for many NMT exercises, which make use of the effects of music on the brain in order to reinforce recovery of speech, movement and cognitive abilities. Other recent examples of accessible instruments include the work of Human Instruments (Matossian and Gehlhaar, 2015), including the Head Space, a head-movement controller for a quadriplegic musician which featured during the 2012 Paralympic Games closing ceremony. An emerging trend in the field of accessible instrument design is the use of Interactive Machine Learning (IML) techniques as a means of adapting DMIs to the varied range of achievable gestures of musicians with disabilities (Katan et al., 2015).

Though physical impairments can prevent users from recreating the same expressive gestures as abled musicians, less immediately apparent expressive gestures can be highlighted through users' constraints. Through a participatory design procedure with a group of musicians with quadriplegic cerebral palsy, McCloskey et al. discovered a rich set of subgestures derived from a simple button press gesture (McCloskey et al., 2015). Though the musicians appeared to be constrained to relatively broad gestures (i.e. pressing a single button), more subtle interaction was achieved through the strength and duration of the button presses.

The guitar has been a popular candidate for instruments in the former category ('therapeutic devices'). Bell made basic adaptations to the guitar including retuning and removing strings in order to make it easier to fret chords (Bell, 2014). Others have explored more complex mechanical adaptations to the guitar. Larsen et al. (2014) developed a guitar adapted for students with hemiplegic cerebral palsy, with a foot-pedal controlled strumming mechanism and open tuning. This arrangement allows for one-handed playing, although the strumming mechanism prevents individual string plucks, and appears to be of a fixed height, position and speed. Larsen also notes that the use of a microcontroller and a digital interface allows for various arrangements to be easily implemented for a range of physical abilities. Another simplified accessible guitar is the 'guitarMasheen' (Meckin and Bryan-kinns, 2013). This was designed for children with learning difficulties taking part in group workshops, and also uses open tunings and simplified plucking mechanisms. In this case, string fretting is also actuated using a solenoid-based system. This allows the children to control the entire guitar through a simplified iPad interface.

The 'Kellycaster', , developed at Drake Music by John Kelly, Gawain Hewitt and Charles Matthews is a guitar-based project that falls into the 'performance-focused instrument' category. The participatory design procedure allowed disabled musician John Kelly to develop an interface for a software guitar that maintains expression over string activation and chord selection, but is suited to his reduced motor functionality. The Kellycaster uses real guitar strings to control a software synthesiser, providing a degree more expression than a simplified keyboard interface, for example.

The goal of the work in this paper is to achieve a fully-realised bass guitar playable with one hand, and so therapeutic adaptations or applications are outside the scope of this article.

\subsection{Approaches to One-Handed Playing}

In this section, we discuss the subgroup of accessible musical instruments that are designed to be played without the use of one hand. Many recent successful one-handed adaptations have come from work done by the One-Handed Musical

\footnotetext{
${ }^{2}$ http://www.drakemusic.org/our-work/research-development/artist-led-projects/john-kelly-the-kellycaster/
} 
Instrument (OHMI) Trust, an organisation which seeks to develop specialist instruments which are both accessible to those with upper-limb disabilities, and capable of facilitating professional or virtuosic performances.

Some of the most successful one-handed instrument adaptations have been wind instruments. This is largely to do with the fact that both hands tend to play a similar role, i.e. pressing different combinations of buttons to open and close valves, while sound activation is achieved using the mouth. This playing method lends itself to a conceptually straightforward (albeit mechanically challenging) adaptation for one hand, whereby the mapping between valve closure and the buttons is reconfigured for a single hand. Examples of successful one-handed wind instrument adaptations can be found on the OHMI website ${ }^{3}$. Snedeker (2005) also summarises several earlier wind instrument adaptations for one hand or otherwise. For many wind instruments, successful one-handed adaptations have been achieved.

String instruments present a more challenging design problem, due to the fact that both hands perform entirely separate roles. Here we refer to the subset of composite chordophones that feature a fingerboard, and strings which can be shortened in order to affect pitch (i.e. not including variants the harp, dulcimer or piano, which feature fixed length pre-tuned strings). These instruments require one hand to clamp the string to the fingerboard at a specific location (note selection), and the other to pluck, bow or otherwise excite the string (note activation). These two tasks are fundamental to string playing and expression; without one, the other would be rendered useless. Fretting is on the surface a relatively simple kinematic procedure, whereas plucking or bowing requires highly accurate placement and pressure, either sustained over a long period of time (bowing), or as an instantaneous gesture (plucking).

Previous approaches to one-handed string playing generally involve coupling note selection and activation processes into a single gesture. This can be achieved by 'tapping' on the fretboard with sufficient force to cause a note to ring out, as seen in Bill Clement's adapted playing style ${ }^{4}$, and the Chapman stick ${ }^{5}$. This allows for highly accomplished performances but produces a timbre that is arguably distinct from a plucked string.

Other approaches involve software synthesis of a string instrument, via physical modelling or high-quality samples, and expressive digital interfaces which allow gestures on multiple axes. One example is the use of the Linnstrument (Linn, 2013), a multi axis controller which provides $\mathrm{X}$ and $\mathrm{Y}$ position as well as pressure data, using buttons arranged in a grid layout similar to fretted stringed instruments. This allows a degree more expression than a standard MIDI keyboard could, making pitch bends, slides, and vibrato possible, in a mode more suited to fretting hand string technique than a keyboard layout provides.

Coupling note selection and activation, either acoustically via a 'tapping' method, or via expressive control of a software synthesiser, essentially reduces a string instrument performance to extended keyboard technique, albeit with the ability to modulate the note after onset. These solutions lack the variety of note onset articulation found on the original instrument.

We suggest that a method of maintaining the separation of note selection and activation would preserve the nature of interaction with a plucked string instrument, and therefore the subtle characteristics of the instrument that come from this interaction. While this could conceivably be achieved with a synthesiser and controller, a mechanical adaptation to a bass guitar would also solve this problem, while preserving the acoustic characteristics of the instrument.

A mechanical adaptation in this context would allow either note selection or activation to be transferred to an alternate limb, in a similar mode to Larsen's actuated guitar (Larsen et al., 2014). This leaves two problems to be solved: the method of mechanical note selection and/or activation; and how these methods are mapped to a one-handed interface. A key design consideration is which hand to 'replace'. This decision has strong implications for the performance of the instrument, not to mention the mechanical complexity of the system.

\subsection{Robotic Stringed Instruments}

The field of musical robotics may provide some inspiration for a mechanical adaptation to the bass guitar. The following section describes automated instruments which are capable of playing back pre-composed pieces of music, however the means of note selection and activation could equally be used in a real-time interactive system.

Kapur (2005) summarises many robotic music instruments, split into piano, audio playback, percussion, string and wind robots, and the various motivations of their creators. For this study, we are particularly interested in the 'plucked bots' subcategory of string robots.

Of the many plucked string instrument robots summarised in Kapur's paper and elsewhere, there appear to be two key approaches to note selection, and two approaches to note activation. Note selection is typically via a movable bridge as

\footnotetext{
${ }^{3}$ http://www.ohmi.org.uk/previous-winning-instruments.html

${ }^{4}$ https://www . youtube. com/watch?v=eskvyuzF1-Y

${ }^{5}$ http://www. stick.com/
} 
in Baginsky's 'Aglaopheme' 6 , a robotic stringed instrument analogous to a slide guitar, or via fixed-position clamping systems analogous to a many-fingered hand performing on a standard guitar.

The fixed-position approach can be used to perform 'hammer-on' gestures, i.e. clamping the string with sufficient force that the note itself rings out (as seen in Jordà's 'Afasia' project (Jordà, 2002)) or to simply change the length of the string at discrete intervals as in typical guitar performance, seen in Squarepusher and Z-machines 'Music for Robots' collaboration ${ }^{7}$.

There are advantages and disadvantages to both approaches to note selection. A moving bridge allows the system to perform continuous pitch variations as in vibrato, slides and pitch bends, but is unable to perform rapid discrete note changes easily (i.e. trills, hammer-ons etc.). Fixed-position systems lack continuous pitch variation but can perform rapid note changes in a way that is slightly more analogous to the human hand. Some machines feature a hybrid system consisting of a moving trolley with separate fixed-position clamps (Compressorheads' robot bassist 'Bones' for instance ${ }^{8}$ ). Such a system could in theory perform many more of the fretting hand gestures of a human player, including both pitch slides and discrete note selection.

Vindriis, McVay and colleagues discuss approaches to an automated bass guitar design, the 'Bassbot', later the 'MechBass' (Vindriis and Carnegie, 2011) (McVay et al., 2011). Vindriis and Carnegie analyse three methods of string plucking using either stepper motor 'pick wheels' or linear solenoid actuators. Proposals for dynamic modulation by adjusting the height of the plucking mechanism are explored. The MechBass incorporates an added damping mechanism using a servo, to prevent strings from ringing out, and to perform muted plucks. Due to the moving bridge design of the MechBass, the strings are spatially separated, and the abundance of motors and actuators requires optical pickups (as opposed to magnetic) to prevent electromagnetic interference. While some amount of expression is achievable using height-adjusted pick wheels and adjustable dampers, the note activation methods afforded by these musical robots are by no means comparable to those offered by the human hand.

Bretan and Weinberg (2016) provide an update to Kapur's summary, with several examples of rather more sophisticated robotic stringed instruments. These include Shibuya's robotic bowing arm for a violin (Shibuya et al., 2012) and Chadefaux's plucking finger for harp (Chadefaux et al., 2012). The latter decribes the attempt to accurately recreate a harp string pluck via mechanical means. Chadefaux et al. attempt to recreate the 'complex mix of displacement, velocity and rotation' seen in the initial conditions of a human-plucked harp string, using a robotic finger which is capable of reproducing the plucking finger's trajectory using two hinges. The robotic finger features a silicon fingertip which approximates the pad of a human fingertip.

\subsection{The Bass Guitar}

The bass guitar is a fretted, plucked stringed instrument. The four strings are typically tuned in 4 ths, from $E_{1}$ to $G_{2}$, the same as a double bass. Basses commonly have between 21 and 24 frets. Common variations include alternate tunings such as drop D or Eb natural, varying number of strings, fretless, and acoustic. The strings are plucked with the fingers and thumb, or with a plectrum.

Electric basses feature heavily in most western pop- and rock-influenced music, and are strongly associated with the rhythm section, although many virtuoso bassists often play 'lead' or melodic lines in these styles. There is some evidence to suggest that the emphasis on rhythm of lower-pitched instruments such as the bass is due to the increased sensitivity in human perception to timing on lower-pitched notes (Hove et al., 2014).

Bass guitars, among other rock band instruments have a recognised 'cultural capital', and are often used in classrooms and music therapy settings due to their recognisability and popularity (Bell, 2014; Westerlund, 2006; Burland and Magee, 2014).

\section{Player Survey}

Designing a bass guitar for one-handed control is likely to involve some compromise: the instrument is ideally suited to two hands, and a mechanical approximation of plucking or fretting hand gestures is likely to be limited in some areas. As well as this, due to the transferral of the interaction from the hands to an alternate limb, we expect to encounter some limitations and compromises in the control method. We designed an online survey in order to establish which elements of bass guitar performance were most important to a bassist's expression and personal style. This would allow us to mitigate against these inherent compromises.

\footnotetext{
${ }^{6}$ http: //www. the-three-sirens.info/

${ }^{7}$ http://warp.net/news/squarepusher-music-for-robots/

${ }^{8}$ https://compressorhead.rocks/
} 


\subsection{Survey Contents}

The survey was divided into three sections: the first section dealt with the individual respondent's self-reported bass playing proficiency, as well as details such as their preferred genre, type of bass guitar, hand dominance and other instruments played. The second section asked respondents to rank ten different performance elements in order of importance. The final section dealt with how users split their practice time between plucking and fretting hand techniques, as well as which hand they felt contributed most to their playing style.

For the second section, we defined 10 key performance elements: rhythmic accuracy, choice of rhythm, choice of note, choice of string, picking style, picking hand articulation, dynamics, fretting hand articulation, timbre of instrument, and use of effects. Respondents were asked to place each element in order of importance by giving each one a rating out of 10 .

The last section comprised of two questions: how much practice is spent on techniques concerned with either the plucking or fretting hand, and which hand is most important in terms of defining the respondent's own playing style. Respondents were invited to give long-form free text answers in order to invite fully-explored insights and not to introduce bias. A final question allowed participants to add any other thoughts that they considered relevant to the study.

\subsection{Results}

48 bassists responded to the survey. Respondents rated their proficiency on a scale from 1 (beginner) to 7 (expert), with an average response of 5.3. The number of years spent playing bass guitar ranged from 2 to 45 years, with an average of 20 years. 35 respondents had been playing bass for at least 10 years. The mean number of years receiving lessons was 2.5 years with a maximum of 10 years. Nearly all respondents performed regularly with an ensemble, spending an average of 5 hours per week (max 22.5 hours) practicing their instrument or in rehearsals.

Respondents were asked which genres they played, mostly answering 'Rock' (67\%), 'Funk' (42\%), 'Jazz' (38\%), 'Pop' (27\%) and 'Blues' (21\%). In total, 36 genres were named, 19 of which were only mentioned once (e.g. 'Psychobilly', 'Cowboy Punk' and '60s pop').

$71 \%$ of respondents played fretted electric 4 -string basses, and $40 \%$ played fretted 5 -string. $10 \%$ played 4-string fretless and $8 \%$ played upright. Other bass types which were mentioned only once included 6-string fretted and fretless, acoustic bass guitar (fretted and fretless), and 3-string fretted (tuned EAD).

The mean score and variance for the importance of each performance element, according to the survey results, is shown in Figure 1. There is a general consensus on the importance of rhythmic accuracy, note choice and rhythm choice, each scoring above 8 out of 10 with a relatively small standard deviation. Picking hand articulation and dynamics appear to be of equal importance, while fretting hand articulation, timbre and picking hand style received similar scores, although the larger standard deviation (between 2.2 and 2.5) suggests less accordance for these factors. String choice scored 4.9 on average, with a standard deviation of 2.4. Respondents seemed to be in agreement on the use of audio effects (i.e. effects pedals or stompboxes) being of the least importance to bass playing style, with a mean score of 2.9 out of 10 .

Figure 2 displays the results for hand importance and practice time. Respondents' rehearsal and practice time was generally split evenly across both hands, with $54 \%$ on the fretting hand and $46 \%$ on the plucking hand. We then asked players which hand was most important to them in terms of their playing style, and invited further comments. 52\% placed most importance on their picking hand, with $23 \%$ for fretting hand, and a further $23 \%$ for both hands. Those who specified their picking hand commented that it was most important for defining their timbre, dynamics and rhythm. Some respondents used terms such as 'feel' and 'groove' when referring to the role of their picking hand. Those who chose their fretting hand mostly commented that their choice of notes, melody and chord were most important to them, which was reflected in their responses to the previous question on performance elements. There was no overt correlation between hand preference and style, ability or years spent playing.

\subsection{Discussion}

Most respondents appeared to agree that rhythmic accuracy was the most important element of bass guitar performance, with a roughly even split between note choice and rhythm choice for second most important. There was far less consensus amongst players over the remaining performance elements. Twice as many respondents said their plucking hand was more important to their style than their fretting hand. Many players acknowledged the difficulty in answering such questions, with several comments referring to the fact that both hands are integral to bass playing. This is perhaps reflected in the fact that the vast majority of players spend equal amounts of time practising with either hand. The results of the survey do suggest that plucking hand artefacts such as rhythm, dynamics and timbre are more important for expression 


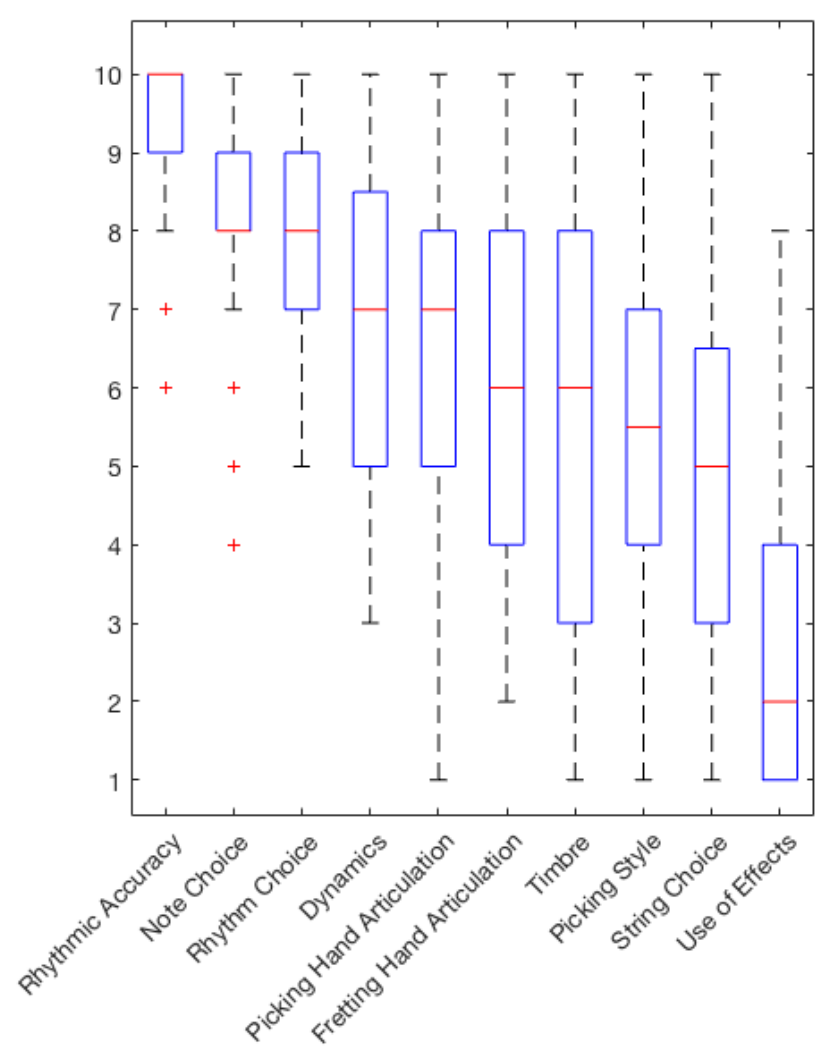

Figure 1: Box plot displaying respondents' order of importance for various bass guitar performance elements

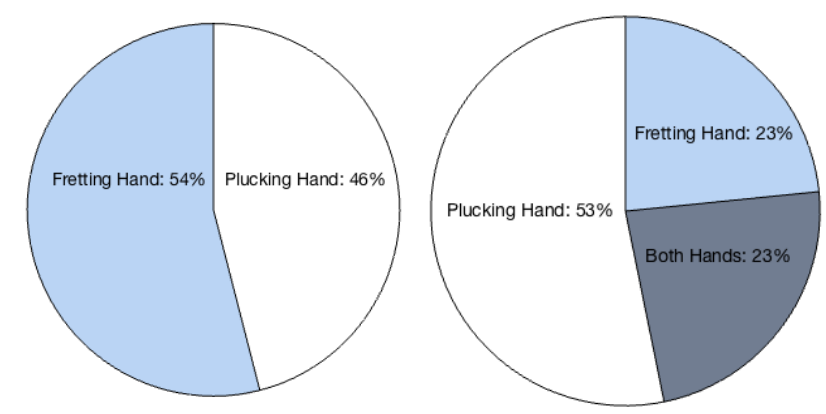

Figure 2: a) Importance of plucking and fretting hand technique to respondents' playing style; b) Amount of practice time focused on plucking and fretting hand techniques

and performance than fretting hand articulation and even note choice. As one player comments: 'you could do so much simply by sticking to the [root notes] and differing where, how and how hard you play the notes'.

This importance placed on rhythm, dynamics and 'groove' suggests that when, where, and how hard the string is plucked is a highly significant factor of bass playing. The pick wheel and solenoid based plucking mechanisms discussed in section 2.3 are not capable of similar degrees of freedom in terms of position, height and pressure as the human hand. We observe that transferring the role of the fretting hand onto an alternate limb would preserve the plucking hand's function, which we hypothesise will preserve the most significant expressive factors of bass playing from our survey.

\section{Design and Implementation}

\subsection{Fretting Mechanism}

In order to test our hypothesis that replacing the fretting hand would preserve the most important aspects of bass performance, we designed a foot-operated fretting mechanism to be attached to the bass. We sought to demonstrate the electromechanical viability of the design and the usability of the interface, in a narrowly focused environment. As such, 
we do not present a completed instrument, but a system suitable for the purposes of a proof-of-concept.

Since the electric bass is designed to be played amplified, replacing the fretting hand for note selection could be achieved either through an electromechanical solution or one based on audio pitch shifting. Digital signal processing methods are flexible and avoid mechanical bulk, but a mechanical fretting system retains the natural relationship between string length and timbre and a direct link between audio and vibrotactivle feedback. Moreover, pitch shifting algorithms typically add latency and, when shifting more than a few semitones, can distort transients. Since our primary goal was to retain the natural action of the plucking hand, we opted for a mechanical approach.

We designed a clamp that could attach to a bass guitar neck, and a system of 'fretting arms', or levers, that are pulled down onto the string when a pull-type solenoid is activated. The fretting arms had to be designed such that they would let remaining strings ring whilst fretting, so a protrusion is added at the location of contact with the string (Figure 3).

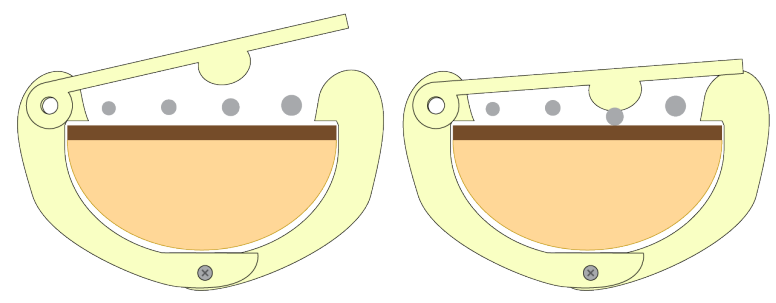

Figure 3: Basic design and functionality for neck clamp and fretting arm, with cross-section of bass guitar neck

We used a mechanical force gauge to determine the minimum force required for a note to ring true when fretted at the 2 nd, 3rd and 4th frets on the A string. This gave an estimate of around $2 \mathrm{~N}$. The height of the strings from the fretboard (action) is around $3 \mathrm{~mm}$. A further $3 \mathrm{~mm}$ is required between the top of the strings and the fretting contact to prevent buzzing from the vibrating string coming into contact with it when not in use. From this, we require a linear motion actuator capable of exerting at least $2 \mathrm{~N}$ of force with a stroke length of at least $6 \mathrm{~mm}$. Pull-type solenoids are ideal for this as they are rapid, strong linear actuators. The user may wish to fret a note for a significant length of time, so larger solenoids are preferred, as they are capable of remaining active for longer periods of time without overheating. For this the BLP Components PED 42-120-611-620 pull-type linear solenoid actuator was chosen, as it is capable of exerting around 4.5N on a continuous duty cycle with a $6 \mathrm{~mm}$ stroke.

Figure 4 shows a schematic of the final fretting mechanism design. We expanded on the neck clamp design to allow for the solenoids to be mounted perpendicular to the fretboard. The ends of the solenoid plungers can then be attached to the tip of the fretter arm. Vertical slots for the solenoid mounting screws allow the height of the solenoid to be adjusted relative to the fretboard. A spring return system prevents the fretting arm from coming into contact with the string. The fretting arm only needs around $6 \mathrm{~mm}$ of clearance from the vibrating string, so its return height is retained by a rod running perpendicular to the neck. We used threaded rods for this purpose as it allows the horizontal position of the neck clamps to be held in place using nuts.

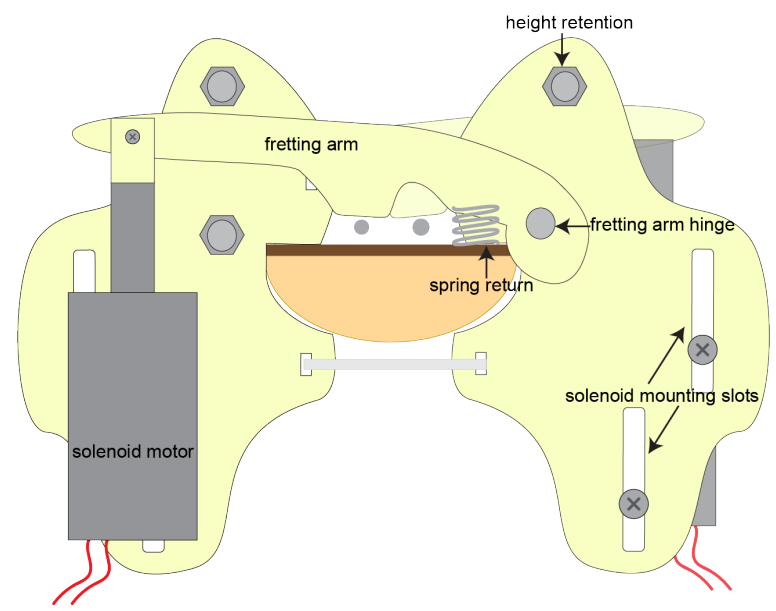

Figure 4: Schematic of final neck clamp design with solenoid mounts, with cross-section of bass guitar neck

As this is a prototype device, we will be covering a limited area of the fretboard, in order to arrive at a proof of concept. We used 6 solenoid motors, with neck clamps at the 2nd, 3rd and 4th frets, providing fretting access to both A and D strings. This allows eight notes to be played, including the open A and D strings: A, B, C, C $\sharp$, D, E, F and F . Figure 5 displays photographs of the completed system attached to the neck. 


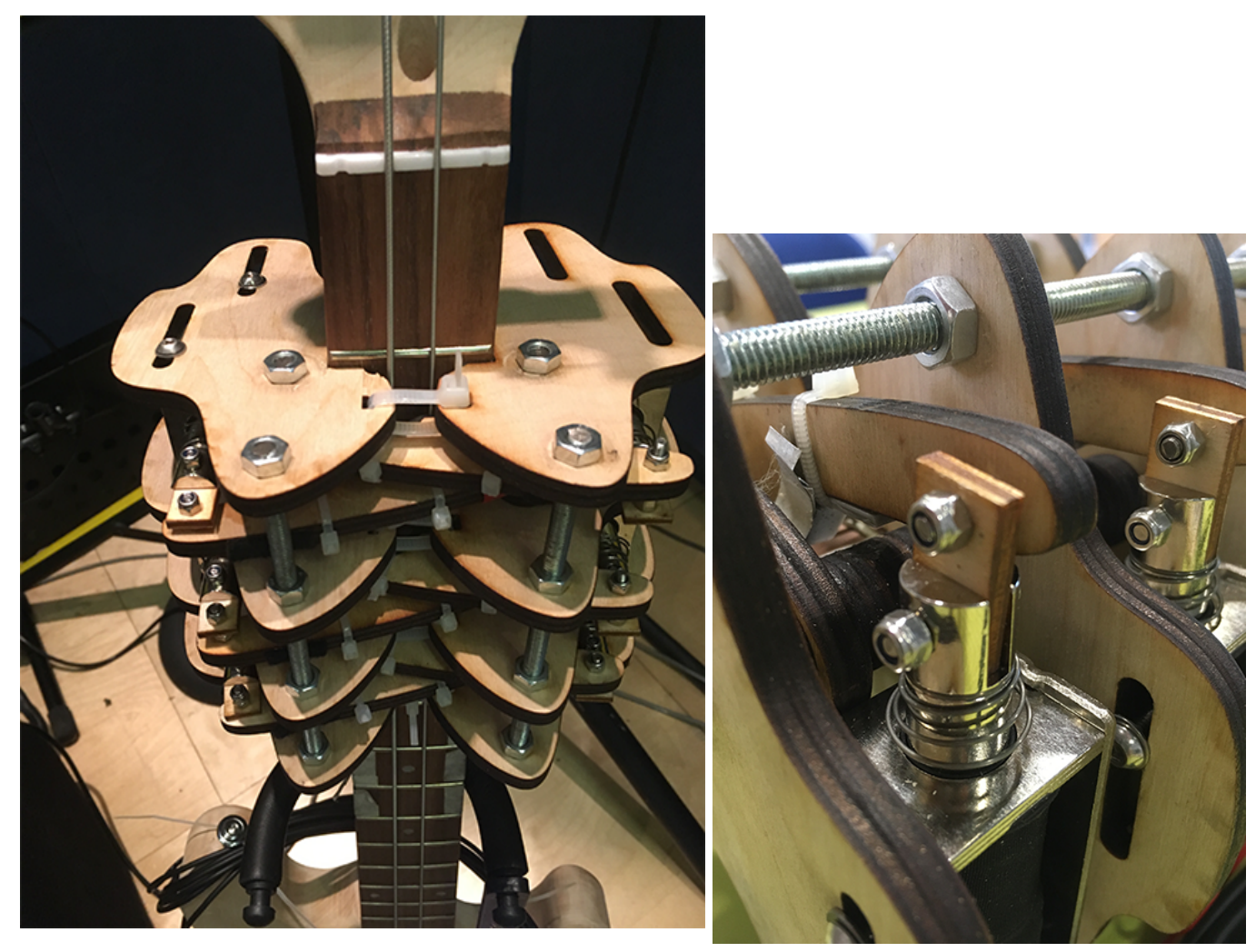

Figure 5: a) side view of neck clamp; b) solenoid plunger attachment to tip of fretting arm

The fretting mechanism uses the Bela platform (McPherson and Zappi, 2015) to drive the solenoid motors and to communicate with the interface. The Bela platform takes MIDI input messages from a USB controller and sets the corresponding pins between $0 \mathrm{~V}$ and $5 \mathrm{~V}$ depending on the control message. The circuit design for a single solenoid channel is shown in Figure 6.

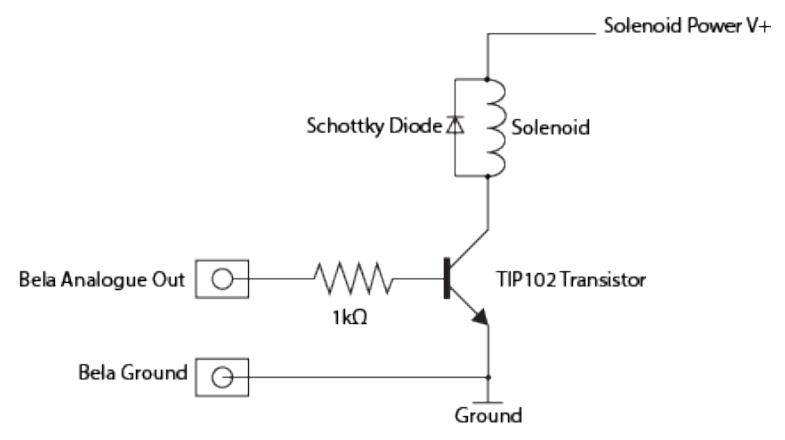

Figure 6: Circuit diagram for a single solenoid channel

\subsection{Interface}

The design problem involved in this project boils down to two key decisions: which hand to 'replace', and which limb or body part to transfer the control of such a system onto. For the Actuated Guitar project, Larsen chooses a foot controller, as the feet and legs are most suited to 'moving in rhythmic patterns for long periods of time' (Larsen et al., 2014). Many assistive technology interfaces make use of eye gaze or head movement (Majaranta, 2011), however this might be suboptimal due to the neck muscle's primary use for stabilising the head, which as Larsen points out, are not suited to prolonged rhythmic movements. Voice control has also been explored for use with enabling devices (Hainisch and Platz, 2007). For this project, we chose to work with foot controllers, but suggest that alternative input methods and devices could be used due to the MIDI-over-USB connectivity. 
We expected to find examples of specialised foot-based interfaces designed for people with upper-limb disabilities who use their feet for everyday tasks, and base our foot controller around such a design. We found instead that in many cases, people with such needs use 'off-the-shelf' devices originally intended to be used with the hands, with little to no adaptations ${ }^{9}$. This may be simply due to a lack of available devices on the market, but suggests that given sufficient motor function of the feet, similar levels of dexterity as the hands is achievable. As a result of this, we opted to use an off-the-shelf MIDI controller as an interface. There exist foot controllers for performing music, which are not necessarily designed for those with disabilities, such as MIDI-enabled organ pedalboards or the Soft Step by Keith McMillen ${ }^{10}$, but we felt that the size and layout of these devices was not appropriate for our eventual design.

The advantage of using a DMI-based interface for our instrument is that we are able to explore various mapping strategies. Unlike acoustic instruments, DMI interfaces are removed from any physical constraints caused by the interface construction, and allow for arbitrary controller layouts. We considered two approaches to the mapping between the controller and the fretting mechanism: 'natural' and 'optimised' mapping. Natural mapping, described by Norman (1990) as 'taking advantage of spatial analogies', is an attempt to make the relationship between the interface and the system as obvious as possible, enabling 'natural' or 'intuitive' control by the user. We define optimised mapping as an approach based on ergonomics: for example arranging note selectors in terms of harmonic relevance in order to minimise foot movement, similar to the layout of the bass keys of an accordion. A thoughtful optimised mapping approach could reduce the size of the required controller and reduce foot movement, but might require 're-learning' of the instrument due to its departure from the fretboard layout.

There are strong arguments for both mapping approaches, and factors such as the available motor function of individual users should be taken into account when considering which approach to use. For the purposes of this study we opted for a natural mapping approach. This would would mean representing the 6 available notes in two rows (representing the A and D strings) and three columns (representing the 2nd, 3rd and 4th frets). We used an Arturia Beatstep ${ }^{11}$, which features two rows of 'drum pads', which are assigned MIDI note numbers by default. The remaining rotary controllers and extra features were not used for this study. Figure 7 displays the mapping strategy used with the Beatstep. Since one of the participants in the following study was left-handed, we used six further drumpads to retain the natural mapping when the bass itself was reversed (the participant was comfortable with the strings themselves being reversed due to experience playing an 'upside-down' right-handed bass).

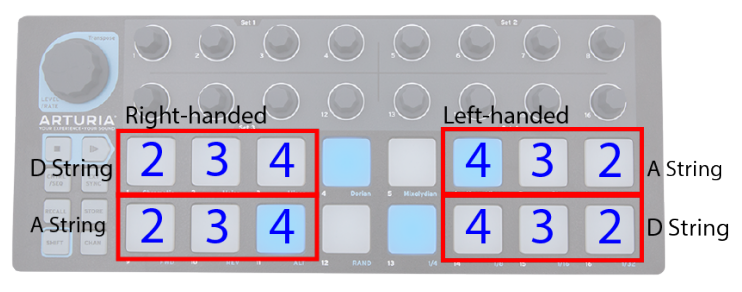

Figure 7: Arturia Beatstep MIDI controller. Annotations describe mapping from drum pads to frets

As seen in section 2.1, many accessible instruments require bespoke designs, highly tailored to the individual's requirements. As such, we do not claim to have come across an ideal approach to mapping. Some users with dextrous use of the feet may prefer a naturally mapped foot controller with a layout resembling that of a bass guitar fretboard. Other users might have very limited movement of any available limbs and might prefer an optimised mapping approach, where minimal movement is required to transition between harmonically relevant notes and chords. There is no clear answer as to which mapping approach is best for this project, and exploring this problem further via a comparative study would provide some valuable insights into adapted instrument design. For the purposes of this project however, we opted for a natural mapping approach.

\subsection{Latency}

McPherson et al. (2016) discuss the importance of latency and jitter in DMIs and measure the audio latency introduced by common prototyping arrangements. For this experiment, we are concerned with the latency between the initial key press on a MIDI controller, and the resultant string being fretted.

\footnotetext{
${ }^{9}$ In particular, author and journalist Sarah Kovac provides video examples of her using her feet to type, apply makeup, and play the piano: http: //sarahkovac.com/popularvideos/

${ }^{10}$ https://www.keithmcmillen.com/products/softstep/

${ }^{11}$ https://ww . arturia.com/beatstep/overview
} 
Figure 8 displays photographs of the experimental setup. We tested the latency of the system by attaching a piezo sensor to the fretboard, directly beneath the fretting arm at the 2nd fret on the D string, and another piezo on the corresponding drum pad on the MIDI controller. We measured the voltages of the two sensors using a two-channel oscilloscope. The delay between the voltage change onset of the two channels gave a good estimate of the latency of the system. We also measured the voltage change of the solenoid line against the two piezo channels. This gave us three separate measurements: overall latency (drumpad piezo $\rightarrow$ fretboard piezo), system latency (drumpad piezo $\rightarrow$ solenoid), and mechanical latency (solenoid $\rightarrow$ fretboard piezo). We repeated the test ten times for each measurement and calculated the average latency, standard deviation and jitter (the amount, in milliseconds, by which the latency varies above and below the mean). The results are displayed in Table 1.
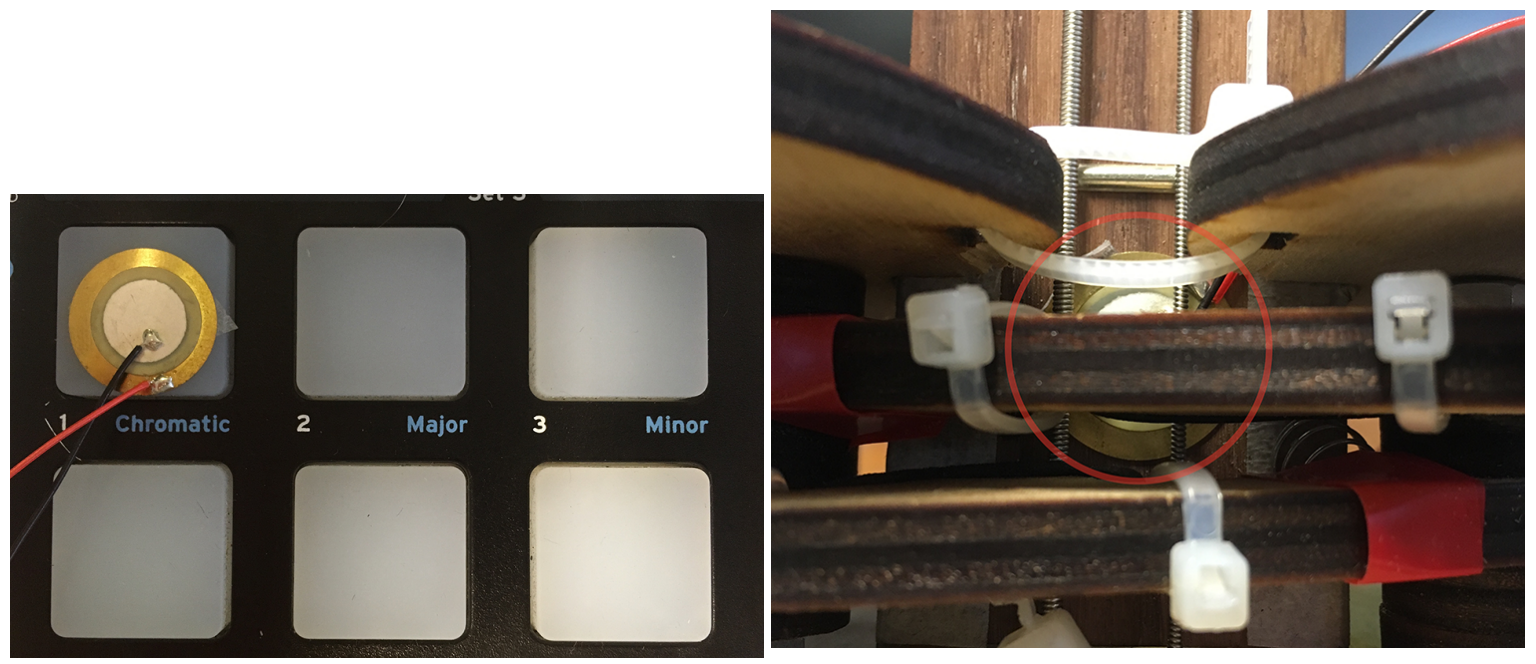

Figure 8: Experimental setup for latency testing: location of the two piezo sensors: a) drumpad, b) fretboard. A third oscilloscope channel measured the voltage change at the output from the corresponding pin on the Bela board.

\begin{tabular}{l|l|l|l} 
Measurement $(\mathrm{ms})$ & Total & Software & Mechanical \\
\hline Mean & 55 & 6 & 50 \\
SD & 0.9 & 0.3 & 0.5 \\
Jitter & \pm 1.6 & \pm 0.5 & \pm 0.8
\end{tabular}

Table 1: Values for three separate latency measurements: total (drumpad piezo $\rightarrow$ fretboard piezo), software (drumpad piezo $\rightarrow$ solenoid), and mechanical (solenoid $\rightarrow$ fretboard piezo)

The overall latency of around $54 \mathrm{~ms}$ is somewhat disappointing when compared with Wessel and Wright's quoted upper limit for latency tolerance of around $10 \mathrm{~ms}$ for DMIs (Wessel and Wright, 2002a). However, Wessel and Wright are concerned primarily with the latency between note activation and the sound being produced. Here, we are measuring the latency between note selection and the corresponding string being fretted, in preparation for plucking. This is a preparatory gesture, that could occur at any point before note onset, and so the latency may be more easily tolerated than with note activation.

Secondly, we posit that the overall latency could be brought down considerably with future iterations of the fretting mechanism. We used basic prototyping tools such as laser-cut plywood and off-the-shelf parts to manufacture the neck attachment. A 3D-printed neck attachment might allow bespoke parts to be made to allow a closer fit to the neck and fretboard, reducing unwanted mechanical movement, as well as weight. Alternative actuators might exist which could provide more speed and control over the fretting mechanism.

\subsection{Subjective Impressions}

The first author (a bass player of 15 years' experience) spent some time practice with the bass in order to provide insight into what to expect from the user study, and to highlight any technical issues. When using the MIDI controller with the free left hand, the interface layout felt intuitive to use. The main difficulty was achieving accuracy when using the feet to select the buttons, while understanding how the buttons related to the fretboard was straightforward. 


\begin{tabular}{l|l|l|lr|l} 
Participant ID & $\begin{array}{l}\text { Hours spent re- } \\
\text { hearsing }\end{array}$ & $\begin{array}{l}\text { Years spent } \\
\text { playing bass }\end{array}$ & $\begin{array}{l}\text { Years spent } \\
\text { playing any } \\
\text { instrument }\end{array}$ & $\begin{array}{l}\text { GoldMSI } \\
\text { melodic dis- } \\
\text { crimination } \\
\text { IQ }\end{array}$ \\
\hline 1 & 4 & 10 & 30 & 106 \\
2 & 2.5 & 16 & 18 & 91 \\
3 & 2 & 15 & 20 & 103 \\
4 & 1.5 & 14 & 22 & 121 \\
5 & 1.5 & 5 & 15 & 103 \\
6 & 2.5 & 17 & 17 & 92
\end{tabular}

Table 2: Study participant profiles

The strength of the solenoids used meant that fretting the strings with the system produced a uniformly strong 'hammeron' sound, unless the string was muted with the plucking hand. A significant amount of mechanical noise was present, although this was less of an issue when playing through an amplifier. The most noticeable effect on playing style was the transferral of muting tasks to the plucking hand.

\section{User Study}

We designed a study in order to assess the performance of the adapted bass and to highlight key design factors to consider for future accessible string instruments. Specifically we wanted to gain more insight into how the transferral of role of the plucking and fretting hands affects bass guitar playing. We were also interested in the efficacy of the natural mapping approach and interface design.

\subsection{Methods}

We conducted a user study comprising six bass players, with a variety of genre preferences and musical experience. They were given access to the adapted bass over three weeks, and asked to rehearse for a minimum of two hours during that time. Participants were asked to compose their own bass accompaniment to a pre defined backing track, with a chord chart for reference (Figure 9). They were also given access to an unadapted bass, with the E and G strings removed, for use when rehearsing and composing their accompaniment.

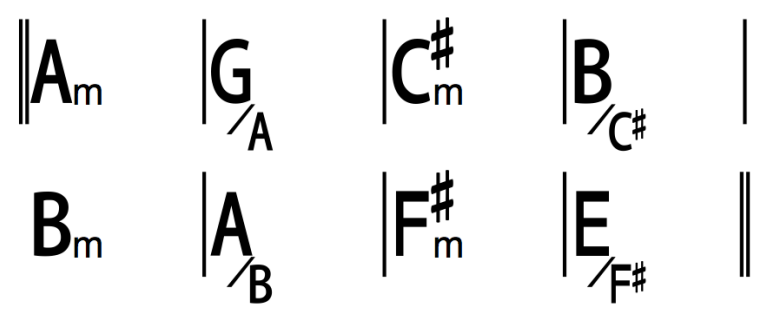

Figure 9: Chord chart provided for backing track accompaniment

Once all six participants had completed their rehearsal sessions, we recorded their performance of the 1-minute long accompaniment. Participants were given as many attempts as they felt necessary in order to perform the piece to a standard they felt reflected their proficiency with the instrument. We recorded audio and video of the performances for annotation.

After the recording was completed, participants filled in a questionnaire dealing with their responses to the bass guitar, as well as asking how much time they had spent with the instrument, and how many years they had been playing bass guitar for. Finally, they were asked to complete the 'melodic discrimination' task from the GoldMSI test battery (Harrison et al., 2016) to provide an indication of each participant's musical proficiency (termed 'melodic discrimination IQ'). Table 2 summarises the participants' musical experience and melodic discrimination IQ.

We annotated each video with respect to four key areas:

Method of note activation: whether notes are plucked or activated using the fretting mechanism (similar to a hammer-on gesture) 
Plucking hand technique: specific techniques employed by the plucking hand including muting the strings to stop notes ringing out, palm muting, playing with a plectrum and tapping on the fretboard

Passing notes: how participants transitioned from one note to another, using open strings or fretted notes.

Foot technique: whether participants used both feet or a single foot, alternated feet for different notes, or lifted the entire foot and replaced it in a different position for consecutive notes.

Finally, we analysed the results from the questionnaire to look for common themes and other salient points from the participant's responses.

\subsection{Results}

\section{Performance Recordings}

Table 3 summarises the annotations made of the performances, in terms of the emergent techniques and gestures observed, and how many participants used them.

\begin{tabular}{|c|c|c|}
\hline Playing Technique & $\begin{array}{l}\text { No. of Partici- } \\
\text { pants }\end{array}$ & Description \\
\hline \multicolumn{3}{|l|}{ Plucking Hand Technique } \\
\hline Plucking hand mute & 4 & Stop notes ringing out using plucking hand \\
\hline $\begin{array}{l}\text { Mechanical hammer-on/pull- } \\
\text { off }\end{array}$ & 2 & Activate note without plucking (using fretting mechanism) \\
\hline Palm-muting with plectrum & 1 & Playing with a plectrum and resting palm on the strings \\
\hline Plucking hand three fingers & 1 & Use of third (ring) finger to alternately pick and damp strings \\
\hline Plucking hand tapping & 1 & $\begin{array}{l}\text { Tapping on the fretboard using plucking hand in order to play notes } \\
\text { higher up the fretboard }\end{array}$ \\
\hline \multicolumn{3}{|r|}{ 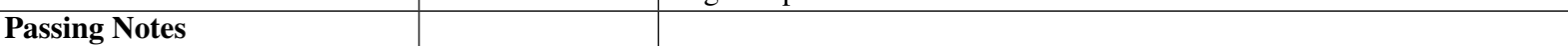 } \\
\hline Open-to-fretted passing note & 6 & Play an open string while transitioning to or from a fretted note. \\
\hline Fretted-to-fretted passing note & 3 & Play a fretted note while transitioning to or from a fretted note \\
\hline \multicolumn{3}{|l|}{ Foot Technique } \\
\hline Foot lift & 6 & Lift entire foot to change fret \\
\hline Single foot & 3 & Use a single foot throughout the performance \\
\hline Double foot & 3 & Use both feet throughout the performance \\
\hline Foot change & 3 & Use alternate foot to change fret \\
\hline Open string position change & 3 & Only change foot/fret position during the sounding of an open string \\
\hline Foot barre & 3 & Use single foot to fret both strings at same fret position \\
\hline
\end{tabular}

Table 3: Emergent playing techniques and number of participants who used them

We noticed that a common feature employed by participants 1,2,3 and 4 was to mute the strings with the plucking hand. This is typically a function of both hands in bass playing, with fretting hand muting usually being employed to shorten the length of individual notes for staccato or rhythmic playing, or to prevent notes from ringing out after skipping strings, in preparation for the next pluck. Instead, functional mutes were transferred to the plucking hand, causing it to take on an additional role.

Two participants made use of the 'mechanical hammer-on' (i.e. the note onset caused by the fretting mechanism activating with sufficient force to cause a note to ring out). These were used in a similar way to a hammer-on played with a hand, i.e. to provide a legato style, and not played on strong beats of the bar.

Participant 5 employed a palm-muted style with a plectrum. This caused every note to be dampened, affecting the timbre and sustain of the note. It appeared that the damping was modulated to allow for some variation in dynamics. Another participant used their ring finger to dampen strings following string skipping, whilst plucking the adjacent string with the index and middle fingers. Perhaps the most unique case was participant 6's use of one-handed tapping with the plucking hand. This involved tapping the fretboard higher up the strings to produce a melody line, whilst changing fret with the fretting mechanism to modulate the bass note. Interestingly, these fret changes did not occur simultaneously with the note onset, but instead occurred during the sounding of the higher 'tapped' notes. 
We examined the use of passing notes during the performances. We define passing notes as notes not played on a strong beat, but which immediately precede or follow a 'strong' note, and may link two such notes together. We observed that all players used the open strings as passing notes between fretted notes on strong beats, on several occasions throughout the performance. Three participants used fretted notes immediately following or preceding another fretted note. There was nothing striking about the harmonic relevance of fretted or open notes, with both being used for strong harmonic notes (i.e. part of the triad of the current chord) or less harmonically related notes.

Three participants used a single foot, while the remaining three used both feet. Those who used a single foot consistently lifted the entire foot and replaced it to change fret position. Those who used both feet alternated between feet to change note, removing the need to lift and replace the entire foot for each new fretted note. Three participants tended to only change the position of their feet during the sounding of an open note, and two occasionally used a single foot to fret two strings at the same fret position.

Participant 4 gave a particularly notable performance, featuring mechanical hammer-ons and pull-offs, and relatively complex syncopated bass lines. We noted a consistency in the dynamics of the performance, with the amplitude of plucked notes being similar to notes caused by mechanical hammer-ons. As well as this, participant 4 displayed greater synchronicity between the actuation of the fretting arms and plucking the corresponding string. There were no occurances of accidental double note onsets, caused by the strong hammer-on from the fretting mechanism sounding a note before the string is plucked. These featured throughout the other performances (except for participant 5, whose use of palm-muting prevented these from occuring).

\section{Questionnaire}

Appendix A lists the questionnaire contents as well as a summary of paraphrased answers across the participants. The responses allowed us to identify participants' thoughts on the playability of common techniques and musical gestures, adaptations to technique, and general responses to the instrument.

The first question asked which gestures typical to a bass guitar would be impossible to produce on the adapted bass. String bends were mentioned three times, dead notes, slides and muting were mentioned twice. Other answers which appeared only once included pull-offs, fast trills, hammer-ons, two handed tapping, legato and sweep picking.

Next, we asked which gestures could be achieved with the adapted bass, given sufficient practice. Two participants said that they could learn to mute the strings with the plucking hand. Answers that could be interpreted as 'playing successive notes quickly' (i.e. 'small runs', 'grace notes', 'fast passages', 'fluid playing') were given three times. Double stopping, legato and staccato playing occured once. One participant stated that most musical gestures typical to bass playing could be achieved with practice, but conceded that fast pieces could be difficult due to lack of precision and speed with the feet.

The third question identified which typical bass guitar gestures felt intuitive to play on the adapted bass, if any. Hammerons were mentioned three times here, although one participant stated that hammer-ons were 'not very controlled' with the instrument. Two participants said that sustained, long notes were easy to play, and another said that tapping on the fretboard with the plucking hand was the same as when playing an unadapted bass.

The final question concerning playability asked if there were any musical gestures that would only be possible to play with the adapted bass. Three participants said there weren't, and the other three were all concerned with the mechanical hammer-ons. One participant said that 'fast mechanical trills' were possible with the adapted bass, and two others stated that the strength of the hammer-on wasn't possible with the hands.

We then asked participants how their plucking hand technique differed from regular bass playing. Three participants commented on the increased focus on muting with the plucking hand, with one stating that the increased level of muting limited the freedom they had with the plucking fingers. One participant commented that the increased concentration on foot placement limited their 'fluidity' with the plucking hand. Another stated that they needed to coordinate their pluck with the button presses more in order to achieve a 'clean sounding note'. The participant who employed the plucking hand tapping technique stated that this did not differ from an unadapted bass.

We invited the respondents to give further comments. One stated their surprise at how quickly they adapted to the limitations of the bass, with dexterity becoming easier over time. Another stated that the instrument felt intuitive to play after an hour's practice.

\subsection{Discussion}

The results from our questionnaire and analysis of the performance recordings highlighted the ways in which modifying a bass guitar in this way can affect a player's technique. 
An unexpected result of the study was the impact that a fretting mechanism such as this has on string muting. A common theme in the questionnaire results was that participants hadn't expected to rely so heavily on their plucking hand to prevent unwanted notes ringing out or accidental activation. This emphasises the secondary role of the fretting hand as a means of muting the strings, by lightly touching the strings rather than clamping them directly onto the fretboard. It appears this is an almost unconscious process when playing bass guitar: only when this secondary role is removed do bass players realise how often they use it. This suggests that muting the strings is not simply a stylistic choice, but a highly functional technique, fundamental to bass guitar playing.

Another system limitation that produced an unexpected effect on playing style was the overall strength of the fretting system. The 'hammer-on' caused by the fretting mechanism produced a considerably loud note, on the same order of magnitude as a plucked note. This could have several knock-on effects. The strength of the note onset caused by the hammer-on sets a lower limit on the strength of plucked notes: this makes softly played passages with hammer-ons quite impossible, as the performer has no control over the strength of the mechanically-triggered notes. Secondly, precise coordination of the fretting mechanism and the plucking hand is required in order to prevent two separate note onsets: the note cannot be fretted silently in preparation for the pluck, so both actions must occur simultaneously. We noted that there was some disagreement amongst participants regarding the playability of hammer-ons with the system. Two participants said they felt intuitive and easy to play, whereas another reported that hammer-ons were impossible. This disagreement could be due to the fact that the latter participant does not consider the idiosyncratic attack and amplitude produced by a mechanical hammer-on to be representative of a typical hammer-on. Another reason for the disagreement could be due to the latency of the system: although a $54 \mathrm{~ms}$ latency might be tolerable for note selection, when used for note onset as a hammer-on this is above the $10 \mathrm{~ms}$ upper limit quoted by Wessel and Wright (2002b).

Participant 5 appeared to mitigate against several of these effects by using a palm-muting technique and plectrum. This prevented unwanted notes from sounding and removed the need to mute each note individually with the plucking hand. This was a stylistically appropriate and convincing use of muting, echoing Motown bassist James Jamerson's use of a piece of foam placed under the strings to reduce sustain, or the heavily palm-muted bass guitar featuring in the introduction to 'Little Green Bag' by the George Baker Selection. Here, the performer used a common stylistic technique as a workaround to mitigate against the limitations of the instrument. The modified functionality of the instrument may have informed the performer's alternative approach to playing.

Participant 4 recreated a typical finger-picked bass performance by adapting his approach to plucking hand muting, as well as maintaining consistent dynamics with the mechanical hammer-ons. The synchronicity between plucking hand and fretting mechanism also prevented any accidental note onsets. Here, the plucking hand technique has been heavily adapted to accommodate the limitations of the fretting mechanism. Participant 5's use of palm-muting evidenced a different approach to these limitations, which affected the timbre and dynamics of the bass. The latter approach requires less re-learning of plucking hand gestures, but limits the dynamic and timbral range of the instrument. The former requires new techniques to be learned, but maintains many of the characteristics of a finger-picked bass performance.

Two participants who used both feet also made use of fretted passing notes. All three participants who used a single foot either only used open strings as passing notes, or created accidental note onsets during fretted passing notes due to the asynchrony between fretting and plucking. Playing an open string in between two fretted notes allows the user to change their foot position during the sounding of the open string, maintaining a fluid performance. A reliance on open passing notes here could suggest an avoidance of rapid foot movements. We suggest that using two feet increases dexterity with the controller and prevents users from being limited to the open strings when changing foot position.

All participants were quick to become accustomed to the naturally mapped interface. This suggests that when designing for musicians who have prior experience with fretted string instruments (for example, someone who has suffered a stroke in later life and no longer has full use of one arm), using an interface layout that is analogous to the fretboard might make 'relearning' the instrument a degree simpler. Many participants noted that the size of the buttons makes individual selection difficult. A bespoke controller should address the ergonomics of foot control, but take into account that larger buttons require a larger controller, and an interface designed for all four strings and up to 24 frets might become prohibitively large to use. The improvement in foot accuracy over a short space of time, backed up by evidence of existing users of similar sized devices, suggests that this is more a matter of experience: over time, users are likely to gain the precision and control required to access the interface with the feet. This in particular is an example of the requirements of the end-user being the most important: while a natural mapping approach makes for a highly intuitive design, an optimised approach might be appropriate for users whose range of motion with the feet is restricted.

\section{Conclusion}

We have demonstrated a prototype method of adaptation for the bass guitar, for players without full use of one arm. Through gaining insight into the key aspects of bass playing, we concluded that preservation of the role of the plucking 
hand would allow users to maintain full control over rhythm and timing, in other words the 'groove'. This approach required the design of an actuated fretting mechanism and controller for an alternate limb. We developed a prototype solenoid-based system which attaches to an unadapted bass guitar neck. This allowed us to explore both the efficacy of this approach for future designs, and the role of the fretting and plucking hands in bass guitar playing. Through a performance study and evaluation of filmed performances, we found several emergent playing techniques that came from the use of an adapted fretting mechanism. Most prominently, the transferral of the string muting tasks to the plucking hand was evident in all of the participants' performances, although with differing approaches.

We conducted the performance study with able-bodied musicians, as our goal was to assess the viability of our approach to one-handed bass playing, and to highlight ways in which it would affect performance. We suggest that future developments of this system, especially where the interface is concerned, should be more informed by the physicality of the intended end-users. The range of potential users of one-handed instruments is vast, from amputees to those with hemiplegia following stroke, and the physical requirements for the interface are likely to be highly variable.

Further iterations of the design and subsequent studies could show the extent to which plucking hand technique can be adapted, and highlight areas of playing technique where mechanical adaptation, rather than modification of technique, is required.

\section{Acknowledgements}

This project was undertaken in collaboration with the One Handed Musical Instrument (OHMI) Trust. It supported by the EPSRC and AHRC Centre for Doctoral Training in Media and Arts Technology under Grant EP/L01632X/1.

\section{References}

Bell, A. P. (2014). Guitars have disabilities: Exploring guitar adaptations for an adolescent with Down syndrome. British Journal of Music Education, 31(3):343-357.

Bretan, M. and Weinberg, G. (2016). A Survey of Robotic Musicianship. Communications of the ACM.

Burland, K. and Magee, W. (2014). Developing identities using music technology in therapeutic settings. Psychology of Music, 42(2):177-189.

Chadefaux, D., Le Carrou, J. L., Vitrani, M. A., Billout, S., and Quartier, L. (2012). Harp plucking robotic finger. IEEE International Conference on Intelligent Robots and Systems, pages 4886-4891.

Hainisch, R. and Platz, M. (2007). Phonetic control: A new approach for continuous, non-invasive device control using the vocal tract. 2007 IEEE 10th International Conference on Rehabilitation Robotics, ICORR'07, 00(c):688-692.

Harrison, P., Collins, T., and Müllensiefen, D. (2016). Assessing melodic discrimination abilities with computerised adaptive testing and automatic item generation. ICAR News.

Hove, M. J., Marie, C., Bruce, I. C., and Trainor, L. J. (2014). Superior time perception for lower musical pitch explains why bass-ranged instruments lay down musical rhythms. Proceedings of the National Academy of Sciences, 111(28):10383-10388.

Hunt, A., Kirk, R., and Neighbour, M. (2004). Interfaces for Music Therapy. IEEE Multimedia.

Jordà, S. (2002). Afasia: the Ultimate Homeric One-man-multimedia-band. Proceedings of the International Conference on New Interfaces for Musical Expression, pages 102-107.

Kapur, A. (2005). A history of robotic musical instruments. Proceedings of the International Computer Music Conference, pages $21-28$.

Katan, S., Grierson, M., and Fiebrink, R. (2015). Using Interactive Machine Learning to Support Interface Development Through Workshops with Disabled People. CHI 2015.

Larsen, J. V., Overholt, D., and Moeslund, T. B. (2014). The actuated guitar: Implementation and user test on children with hemiplegia. In NIME '14 Proceedings of the 2014 Conference on New Interfaces for Musical Expression, pages $60-65$.

Larsen, J. V., Overholt, D., and Moeslund, T. B. (2016). The Prospects of Musical Instruments For People with Physical Disabilities. NIME '16 Proceedings of the 2016 Conference on New Interfaces for Musical Expression, pages 327-331. 
Lin, H. W. J., Aflatoony, L., and Wakkary, R. (2014). Design for one: A game controller for a quadriplegic gamer. Conference on Human Factors in Computing Systems - Proceedings, pages 1243-1248.

Linn, R. (2013). LinnStrument and other new expressive musical controllers. The Journal of the Acoustical Society of America, 134(5):4053-4053.

Majaranta, P. (2011). Gaze Interaction and Applications of Eye Tracking: Advances in Assistive Technologies: Advances in Assistive Technologies.

Matossian, V. and Gehlhaar, R. (2015). Human Instruments: Accessible Musical Instruments for People with Varied Physical Ability. Studies in health technology and informatics, 219:202-207.

McCloskey, B., Bridges, B., and Lyons, F. (2015). Accessibility and dimensionality : enhanced real time creative independence for digital musicians with quadriplegic cerebral palsy. pages 24-27.

McPherson, A. P., Jack, R. H., and Moro, G. (2016). Action-Sound Latency: Are Our Tools Fast Enough? Proceedings of the International Conference on New Interfaces for Musical Expression.

McPherson, A. P. and Zappi, V. (2015). An environment for submillisecond-latency audio and sensor processing on BeagleBone Black. Audio Eng. Soc. Conv. 138. Audio Engineering Society, 2015., pages 4-9.

McVay, J., Carnegie, D. A., Murphy, J., and Kapur, A. (2011). MechBass: A Systems Overview of a New Four- Stringed Robotic Bass Guitar.

Meckin, D. and Bryan-kinns, N. (2013). moosikMasheens : Music , Motion and Narrative with Young People who have Complex Needs. Idc 2013, pages 66-73.

Nayak, S., Wheeler, B. L., Shiflett, S. C., and Agostinelli, S. (2000). Effect of music therapy on mood and social interaction among individuals with acute traumatic brain injury and stroke. Rehabilitation Psychology, 45(3):274-283.

Norman, D. (1990). Design of Everyday Things.

Särkämö, T., Tervaniemi, M., Laitinen, S., Forsblom, A., Soinila, S., Mikkonen, M., Autti, T., Silvennoinen, H. M., Erkkilä, J., Laine, M., Peretz, I., and Hietanen, M. (2008). Music listening enhances cognitive recovery and mood after middle cerebral artery stroke. Brain, 131(3):866-876.

Shibuya, K., Ideguchi, H., Ikushima, K., Cho, J., Kato, S., Kanoh, M., Ito, H., Hashimoto, S., Inokuchi, S., Kajitani, M., Konczak, J., Velden, H., Jaeger, L., Kusuda, Y., Sakamoto, D., Ishiguro, H., Shibuya, K., Matsuda, S., Sobh, T. M., Wange, B., Solis, J., Ninomiya, T., Petersen, K., Takeuchi, M., Takanishi, A., Solis, J., Taniguchi, K., Ninomiya, T., Petersen, K., Yamamoto, T., and Takanishi, A. (2012). Volume Control by Adjusting Wrist Moment of Violin-Playing Robot. International Journal of Synthetic Emotions, 3(2):31-47.

Snedeker, J. P. (2005). Adaptive engineering for musical instruments. Medical Problems of Performing Artists, 20(2):8999.

Swingler, T. (1998). The invisible keyboard in the air : An overview of the educational, therapeutic and creative applications of the EMS Soundbeam ${ }^{\text {TM }}$. 2nd Euro. Conf. Disability, Virtual Reality \& Assoc. Tech, pages 253-259.

Vindriis, R. G. and Carnegie, D. A. (2011). A Comparison of Pick-Based Strategies for Robotic Bass Playing. 1:21-22.

Wessel, D. and Wright, M. (2002a). Problems and Prospects for Intimate Musical Control of Computers. Computer Music journal, 26(3):11-14.

Wessel, D. and Wright, M. (2002b). Problems and Prospects for Intimate Musical Control of Computers. Computer Music journal, 26(3):11-14.

Westerlund, H. (2006). Garage rock bands: a future model for developing musical expertise? International Journal of Music Education, 24(2):119-125. 


\section{A Questionnaire Responses}

Q1: Were there any musical gestures that you regularly use when playing bass guitar, that you felt were impossible to play on the one-handed bass?

- 'The range of melodic movement was hindered by the number of string and frets. No ability to bend of move the strings after initial onset.'

- 'Ordinarily I use my fretting hand to slide up to notes and also to bend the strings. Ifound this impossible to achieve on the instrument. I also found through playing the instrument how often I use my fretting hand to deaden notes on the neck of the guitar. I hadn't realised that I did this so frequently, and it was not possible to achieve this with the instrument.'

- 'bends, pull-offs, fast trills'

- 'Muting the notes was the hardest part. Slides and more larger note range also were missing.'

- 'hammer ons, dead notes'

- 'Two hands tapping, fast licks with legato and sweep picking'

Q2: Were there any musical gestures that you regularly use when playing bass guitar, that you felt were possible, with a lot of practice, to play on the one-handed bass?

- 'I started to mute with the right hand by week two. This was [hard] in week 1.'

- '[I like to] use gracenotes, and small runs between notes. The fluidity of playing did increase with time - during my initial run through I was not able to to do this at all, but by the end of the practice I was able to roughly approximate this. I also often fret two notes while playing. This was tricky for some notes when playing ... however with further practice this might be possible by using two feet to control the fretting.'

- 'More right hand techniques require a lot more practice as you need to mute the bass completely with this hand. Hammer-ons required a lot more practice to get accurate. Playing legato takes a lot more practice, as well as playing stacatto. Palm muting requires a strap or other form of stabilisation for the bass - same with any slap/pops'

- 'Hammer ons (but I'm not using them too often).'

- 'fast passages'

- '... with time I could play most of musical gestures. However, for pure mechanical reasons, toes cannot be as fast as precise as the fingers thus $i$ won't expect somebody to be able to play fast pieces with this instrument, even if an ad-hoc pedalboard is designed.'

Q3: Were there any musical gestures that you regularly use when playing bass guitar, that you felt were easy or intuitive to play on the one-handed bass?

- 'hammer ons were possible with the device, however not very controlled.'

- 'Something I regularly use is hammer on and pull off. The way the instrument was constructed made hammer on particularly achievable, and was a very intuitive technique to use. Pull off was implied in the way the strings were fretted, but it wasn't as intuitive.'

- 'No'

- 'sustained - re-triggered notes'

- 'tapping with my right hand. And also long notes are quite easy'

Q4: Were there any musical gestures that you used with the one-handed bass, that would be impossible on a regular bass guitar?

- 'No' [three participants]

- 'Perhaps the strength of the hammer on / fretting was something I couldn't necessarily achieve with a regular instrument'

- 'Very fast mechanical trills. I did try to add these to the groove, but they require more practicing.'

- 'The very sharp hit of the hammers results in a idiosyncratic attack that is not doable with a regular bass. Other than that, in this current prototype, I don't think so. However, I think that the highest limitation is the foot controller. Maybe it worth exploring how to operate the hammers with other body parts - not sure which though' 
Q5: When playing the one-handed bass, how did your plucking hand technique differ from your regular bass playing?

- 'I needed to incorporate mutes and stops with the right hand. These were usually done with the left. This additional muting limited the freedom I had with the two fingers. Limitations on string also hindered usual movements to the 5th or octave.'

- 'As I was playing the instrument upside down there wasn't a natural place to rest my thumb, so this did influence my plucking hand position. I found at the beginning of the practice when plucking the strings my fluidity was impeded as I was thinking quite a lot about the positioning of my feet ... By the end I think I had the same level of fluidity with plucking that I would normally have.'

- 'Yes, it was more focused on muting. I also needed to coordinate my pluck more with the buttons presses for fretting as I to get a clean sounding note the string ideally needed to be muted when the fretting button was pressed, then plucked.'

- 'Very different muting technique. I used my 3rd finger to mute the upper string a lot. When playing steady eighteen muted notes each finger (2 and 3) muted the string immediately after the other finger plucked.'

- 'having less strings makes the hand rest in a different way, so not easy to do palm-muting.'

- 'I did not differ'

\section{Notable comments:}

- 'I was surprised how quickly the I adapted to some of the limitations. Although dexterity and playing became easier, the lack of note range was an ongoing limitation.

- 'After practicing with the instrument for around an hour I found it quite intuitive to play. Obviously due to the limitations of the notes available to play this did change the way I approached the piece of music, but I felt comfortable playing the instrument overall.'

- 'Trying different layouts for the foot controller will be interesting.'

- 'If I had to spend more time with this I would surely write ad-hoc scores in which I have 2 separate staffs, one for the hand and one for the feet. I'd probably write a "fingering" notation for the feet too, as sometimes I was using the left big toe and other times the right one.'

\section{Practical considerations:}

- Weight of neck makes playing uncomfortable

- Electronics attached to body of bass obstruct plucking hand especially left handed playing

- Reduced number of strings affects palm muting

- Plucking hand and arm also used for stabilisation, making common techniques harder/uncomfortable/impossible (i.e. slapping and popping)

- Size of buttons makes playing with feet difficult

- Responsiveness for rapid repeated button presses requires improvement

- Additional 'function' buttons on the foot controller were often selected by accident 